\title{
GENERAL OPIAL TYPE INEQUALITY FOR QUOTIENT OF FUNCTIONS
}

\author{
ANA BARBIR, KRISTINA KRULIĆ HIMMELREICH AND JOSIP PEČARIĆ
}

\begin{abstract}
In this paper we give the general Opial type inequality for quotient of functions. Our result contains two functions, convex and concave functions. We prove a new general inequality on a measure space $(\Omega, \Sigma, \mu)$. We apply our result to numerous symmetric functions and obtain new results that involve Green's functions, Lidstone series and the Hermite's interpolating polynomials.
\end{abstract}

\section{INTRODUCTION}

In 1960, Opial [5] proved the following inequality:

Let $f \in C^{1}[0, h]$ be such that $f(0)=f(h)=0$ and $f(x)>0$ for $x \in(0, h)$. Then

$$
\int_{0}^{h}\left|f(x) f^{\prime}(x)\right| d x \leq \frac{h}{4} \int_{0}^{h}\left[f^{\prime}(x)\right]^{2} d x,
$$

where $h / 4$ is the best possible.

This inequality has been generalized and extended in several directions (for more details see e.g [1], [3]).

In the sequel let $(\Omega, \Sigma, \mu)$ be a measure space and let $k: \Omega \times \Omega \rightarrow \mathbb{R}$ be a symmetric nonnegative or nonpositive function such that

$$
K(x):=\int_{\Omega} k(x, y) d \mu(y), K(x) \neq 0, \text { a.e. } x \in \Omega,
$$

such that $|K(x)|<\infty$. In the rest of the paper we assume that all integrals are well defined. The following result is given in [4].

Theorem 1.1. Let $k: \Omega \times \Omega \rightarrow \mathbb{R}$ be a symmetric nonnegative or nonpositive function. If $f$ is a positive convex function, $g$ a positive concave

2010 Mathematics Subject Classification. Primary 26D15, Secondary 26A51.

Key words and phrases. Opial inequality, Green function, Jensen inequality, Lidstone polynomial, Hermite interpolating polynomial.

Copyright (c) 2016 by ANUBIH. 
function on an interval $I \subseteq \mathbb{R}, v: \Omega \rightarrow \mathbb{R}$ is either nonnegative or nonpositive, such that $\operatorname{Im}|v| \subseteq I$ and $u$ defined by

$$
u(x):=\int_{\Omega} k(x, y) v(y) d \mu(y)<\infty,
$$

then the following inequality

$$
\int_{\Omega}|K(x)| f\left(\left|\frac{u(x)}{K(x)}\right|\right) g(|v(x)|) d \mu(x) \leq \int_{\Omega}|K(x)| f(|v(x)|) g\left(\left|\frac{u(x)}{K(x)}\right|\right) d \mu(x)
$$

holds, where $K$ is defined by (1.2).

The obtained inequalities are not direct generalizations of the Opial inequality but are of Opial type because the integrals contain function and its integral representation. In this paper we give a generalization of inequality (1.4).

\section{The MAIN RESUlts}

Our first result is given in the next theorem.

Theorem 2.1. Let $k: \Omega \times \Omega \rightarrow \mathbb{R}$ be a symmetric non-negative or nonpositive function and $K$ be defined by (1.2). If $f$ is a positive convex function, $g$ a positive concave function on an interval $I \subseteq \mathbb{R}, v, n: \Omega \rightarrow \mathbb{R}$ are either non-negative or non-positive, such that $\operatorname{Im}|v|, \operatorname{Im}\left|\frac{v}{n}\right| \subseteq I$, u defined by

$$
u(x):=\int_{\Omega} k(x, y) v(y) d \mu(y)<\infty,
$$

and $m$ defined by

$$
m(x):=\int_{\Omega} k(x, y) n(y) d \mu(y)<\infty,
$$

then the following inequality

$$
\begin{aligned}
\int_{\Omega}|m(x)| f\left(\left|\frac{u(x)}{m(x)}\right|\right) g(|v(x)|) d \mu(x) \\
\leq \int_{\Omega}|n(x)||K(x)| f\left(\left|\frac{v(x)}{n(x)}\right|\right) g\left(\left|\frac{u(x)}{K(x)}\right|\right) d \mu(x)
\end{aligned}
$$

holds, where $K$ is defined by (1.2). 
Proof. By using Jensen's inequality and the Fubini theorem we find that

$$
\begin{aligned}
\int_{\Omega}|m(x)| f\left(\left|\frac{u(x)}{m(x)}\right|\right) g(|v(x)|) d \mu(x) \\
\quad=\int_{\Omega}|m(x)| f\left(\frac{\left|\int_{\Omega} k(x, y) v(y) d \mu(y)\right|}{|m(x)|}\right) g(|v(x)|) d \mu(x) \\
\leq \int_{\Omega}|m(x)| f\left(\int_{\Omega} \frac{|k(x, y) n(y)|}{|m(x)|} \frac{|v(y)|}{|n(y)|} d \mu(y)\right) g(|v(x)|) d \mu(x) \\
\leq \int_{\Omega}\left(\int_{\Omega}|k(x, y) n(y)| f\left(\frac{|v(y)|}{|n(y)|}\right) d \mu(y)\right) g(|v(x)|) d \mu(x) \\
=\int_{\Omega} f\left(\frac{|v(y)|}{|n(y)|}\right)|n(y)|\left(\int_{\Omega}|k(x, y)| g(|v(x)|) d \mu(x)\right) d \mu(y) .
\end{aligned}
$$

Since $k$ is a symmetric function we get that $|k(x, y)|=|k(y, x)|$ so by using Jensen's inequality we obtain that

$$
\begin{aligned}
& \int_{\Omega} f\left(\frac{|v(y)|}{|n(y)|}\right)|n(y)|\left(\int_{\Omega}|k(y, x)| g(|v(x)|) d \mu(x)\right) d \mu(y) \\
& \leq \int_{\Omega}|n(y)| f\left(\frac{|v(y)|}{|n(y)|}\right)|K(y)| g\left(\frac{1}{|K(y)|} \int_{\Omega}|k(y, x) v(x)| d \mu(x)\right) d \mu(y) \\
& =\int_{\Omega}|n(y)| f\left(\frac{|v(y)|}{|n(y)|}\right)|K(y)| g\left(\frac{|u(y)|}{|K(y)|}\right) d \mu(y)
\end{aligned}
$$

and the proof is complete.

Remark 2.1. If $n(y)=1$, then $m(x)=K(x)$ and inequality (2.4) reduces to (1.4).

We apply our result to numerous symmetric functions and obtain new results that involve Green's functions, Lidstone series and the Hermite's interpolating polynomials.

Consider the Green function $G$ defined on $[a, b] \times[a, b]$ by

$$
G(t, s)= \begin{cases}\frac{(t-b)(s-a)}{b-a}, & a \leq s \leq t ; \\ \frac{(t-a)(s-b)}{b-a}, & t \leq s \leq b .\end{cases}
$$

The function $G$ is convex under $s$, it is symmetric non-positive function and it is also convex under $t$. It is continuous under $s$ and continuous under $t$. For any function $\varphi:[a, b] \rightarrow \mathbb{R}, \varphi \in C^{2}([a, b])$, we can easily show by integrating by parts that the following is valid

$$
\varphi(x)=\frac{b-x}{b-a} \varphi(a)+\frac{x-a}{b-a} \varphi(b)+\int_{a}^{b} G(x, s) \varphi^{\prime \prime}(s) d s,
$$


where the function $G$ is defined as above in (2.4). Since function $G$ is a non-positive symmetric function we can apply Theorem 2.1 and obtain the following corollary.

Corollary 2.1. If $f$ is a positive convex function and $g$ a positive concave function on an interval $I \subseteq \mathbb{R}$, then the inequality

$$
\begin{aligned}
\int_{a}^{b} \mid m(x)-\frac{b-x}{b-a} & m(a)-\frac{x-a}{b-a} m(b) \mid \\
& \times f\left(\frac{\left|\varphi(x)-\frac{b-x}{b-a} \varphi(a)-\frac{x-a}{b-a} \varphi(b)\right|}{\left|m(x)-\frac{b-x}{b-a} m(a)-\frac{x-a}{b-a} m(b)\right|}\right) g\left(\left|\varphi^{\prime \prime}(x)\right|\right) d x \\
\leq \frac{1}{2} \int_{a}^{b}\left|m^{\prime \prime}(x)\right|(b & -x)(x-a) f\left(\frac{\left|\varphi^{\prime \prime}(x)\right|}{\left|m^{\prime \prime}(x)\right|}\right) \\
& \times g\left(\frac{2\left|\varphi(x)-\frac{b-x}{b-a} \varphi(a)-\frac{x-a}{b-a} \varphi(b)\right|}{(b-x)(x-a)}\right) d x
\end{aligned}
$$

holds for all functions $\varphi:[a, b] \rightarrow \mathbb{R}, \varphi \in C^{2}([a, b])$ such that $\varphi^{\prime \prime}$ is either non-negative or non-positive function and $m:[a, b] \rightarrow \mathbb{R}$ such that $m^{\prime \prime}$ is either non-negative or non-positive function.

Proof. Since function $G$ defined by (2.4) is a non-positive symmetric function we can apply Theorem 2.1. Let $\Omega=[a, b], k(x, s)=G(x, s), v(s)=$ $\varphi^{\prime \prime}\left(s, n(x)=m^{\prime \prime}(x)\right.$. Then

$$
\begin{gathered}
u(x)=\int_{a}^{b} G(x, s) \varphi^{\prime \prime}(s) d s=\varphi(x)-\frac{b-x}{b-a} \varphi(a)-\frac{x-a}{b-a} \varphi(b), \\
|K(x)|=\int_{a}^{b}|G(x, s)| d s=\frac{(b-x)(x-a)}{2} \\
m(x)=\int_{a}^{b} G(x, s) m^{\prime \prime}(s) d s=m(x)-\frac{b-x}{b-a} m(a)-\frac{x-a}{b-a} m(b)
\end{gathered}
$$

and inequality (2.3) becomes

$$
\begin{aligned}
& \int_{a}^{b}\left|m(x)-\frac{b-x}{b-a} m(a)-\frac{x-a}{b-a} m(b)\right| \\
& \times f\left(\frac{\left|\varphi(x)-\frac{b-x}{b-a} \varphi(a)-\frac{x-a}{b-a} \varphi(b)\right|}{\left|m(x)-\frac{b-x}{b-a} m(a)-\frac{x-a}{b-a} m(b)\right|}\right) g\left(\left|\varphi^{\prime \prime}(x)\right|\right) d x \\
& \leq \frac{1}{2} \int_{a}^{b}\left|m^{\prime \prime}(x)\right|(b-x)(x-a) f\left(\frac{\left|\varphi^{\prime \prime}(x)\right|}{\left|m^{\prime \prime}(x)\right|}\right)
\end{aligned}
$$




$$
\times g\left(\frac{2\left|\varphi(x)-\frac{b-x}{b-a} \varphi(a)-\frac{x-a}{b-a} \varphi(b)\right|}{(b-x)(x-a)}\right) d x
$$

so the proof is complete.

Remark 2.2. If $\varphi(a)=\varphi(b)=0$, then (2.7) reduces to

$$
\begin{aligned}
& \int_{a}^{b} \mid m(x)- \frac{b-x}{b-a} m(a)-\frac{x-a}{b-a} m(b) \mid \\
& \times f\left(\frac{\mid \varphi(x \mid}{\left|m(x)-\frac{b-x}{b-a} m(a)-\frac{x-a}{b-a} m(b)\right|}\right) g\left(\left|\varphi^{\prime \prime}(x)\right|\right) d x \\
& \leq \frac{1}{2} \int_{a}^{b}\left|m^{\prime \prime}(x)\right|(b-x)(x-a) f\left(\frac{\left|\varphi^{\prime \prime}(x)\right|}{\left|m^{\prime \prime}(x)\right|}\right) g\left(\frac{2|\varphi(x)|}{(b-x)(x-a)}\right) d x
\end{aligned}
$$

Remark 2.3. If $m^{\prime \prime}(x)=1$, then $m(x)=K(x)$ and (2.7) reduces to

$$
\begin{aligned}
& \int_{a}^{b}(b-x)(x-a) f\left(\frac{2\left|\varphi(x)-\frac{b-x}{b-a} \varphi(a)-\frac{x-a}{b-a} \varphi(b)\right|}{(b-x)(x-a)}\right) g\left(\left|\varphi^{\prime \prime}(x)\right|\right) d x \\
& \leq \int_{a}^{b}(b-x)(x-a) f\left(\left|\varphi^{\prime \prime}(x)\right|\right) g\left(\frac{2\left|\varphi(x)-\frac{b-x}{b-a} \varphi(a)-\frac{x-a}{b-a} \varphi(b)\right|}{(b-x)(x-a)}\right) d x
\end{aligned}
$$

that is given in [4].

We continue with the definition of Lidstone series. Lidstone series is a generalization of Taylor's series. It approximates to a given function in the neighborhood of two points (instead of one). Such series have been studied by G. J. Lidstone (1929), H. Poritsky (1932), J. M. Wittaker (1934) and others.

Definition 2.1. Let $\varphi \in C^{\infty}([0,1])$, then Lidstone series has the form

$$
\sum_{k=0}^{\infty}\left(\varphi^{(2 k)}(0) \Lambda_{k}(1-x)+\varphi^{(2 k)}(1) \Lambda_{k}(x)\right),
$$

where $\Lambda_{n}$ is a polynomial of degree $2 n+1$ defined by the relations

$$
\begin{aligned}
& \Lambda_{0}(t)=t \\
& \Lambda_{n}^{\prime \prime}(t)=\Lambda_{n-1}(t), \\
& \Lambda_{n}(0)=\Lambda_{n}(1)=0, n \geq 1 .
\end{aligned}
$$


Another explicit representations of Lidstone polynomial are given by [2] and $[6]$,

$$
\begin{aligned}
\Lambda_{n}(t) & =(-1)^{n} \frac{2}{\pi^{2 n+1}} \sum_{k=1}^{\infty} \frac{(-1)^{k+1}}{k^{2 n+1}} \sin k \pi t \\
\Lambda_{n}(t) & =\frac{1}{6}\left[\frac{6 t^{2 n+1}}{(2 n+1) !}-\frac{t^{2 n-1}}{(2 n-1) !}\right] \\
& -\sum_{k=0}^{n-2} \frac{2\left(2^{2 k+3}-1\right)}{(2 k+4) !} B_{2 k+4} \frac{t^{2 n-2 k-3}}{(2 n-2 k-3) !}, n=1,2, \ldots, \\
\Lambda_{n}(t) & =\frac{2^{2 n+1}}{(2 n+1) !} B_{2 n+1}\left(\frac{1+t}{2}\right), n=1,2 \ldots
\end{aligned}
$$

where $B_{2 k+4}$ is the $(2 k+4)$-th Bernoulli number and $B_{2 n+1}\left(\frac{1+t}{2}\right)$ is a Bernoulli polynomial.

In [7], Widder proved the fundamental lemma:

Lemma 2.1. If $\varphi \in C^{(2 n)}([0,1])$, then

$$
\varphi(t)=\sum_{k=0}^{n-1}\left[\varphi^{(2 k)}(0) \Lambda_{k}(1-t)+\varphi^{(2 k)}(1) \Lambda_{k}(t)\right]+\int_{0}^{1} G_{n}(t, s) \varphi^{(2 n)}(s) d s
$$

where

$$
G_{1}(t, s)=G(t, s)= \begin{cases}(t-1) s, & \text { if } s \leq t \\ (s-1) t, & \text { if } t \leq s\end{cases}
$$

is the homogeneous Green's function of the differential operator $\frac{d^{2}}{d s^{2}}$ on $[0,1]$, and with the successive iterates of $G(t, s)$

$$
G_{n}(t, s)=\int_{0}^{1} G_{1}(t, p) G_{n-1}(p, s) d p, n \geq 2 .
$$

Lidstone polynomial can be expressed, in terms of $G_{n}(t, s)$ as

$$
\Lambda_{n}(t)=\int_{0}^{1} G_{n}(t, s) s d s
$$

Notice that $G_{n}(t, s)$ is a symmetric function. Now we give the following special case of Theorem 1.1.

Corollary 2.2. Let $\varphi:[a, b] \rightarrow \mathbb{R}$ be of $C^{(2 n)}$ on $[a, b], n \geq 1$ such that $\varphi^{(2 n)}$ is either non-negative or non-positive function and $m:[a, b] \rightarrow \mathbb{R}$ be of $C^{(2 n)}$ on $[a, b], n \geq 1$ such that $m^{(2 n)}$ is either non-negative or non-positive 
function. If $f$ is a positive convex function and $g$ a positive concave function on an interval $I \subseteq \mathbb{R}$, then the inequality

$$
\begin{aligned}
& \int_{a}^{b}\left|m(x)-\sum_{k=0}^{n-1}(b-a)^{2 k}\left[m^{(2 k)}(a) \Lambda_{k}\left(\frac{b-x}{b-a}\right)+m^{(2 k)}(b) \Lambda_{k}\left(\frac{x-a}{b-a}\right)\right]\right| \\
& \times f\left(\frac{\left|\varphi(x)-\sum_{k=0}^{n-1}(b-a)^{2 k}\left[\varphi^{(2 k)}(a) \Lambda_{k}\left(\frac{b-x}{b-a}\right)+\varphi^{(2 k)}(b) \Lambda_{k}\left(\frac{x-a}{b-a}\right)\right]\right|}{\left|m(x)-\sum_{k=0}^{n-1}(b-a)^{2 k}\left[m^{(2 k)}(a) \Lambda_{k}\left(\frac{b-x}{b-a}\right)+m^{(2 k)}(b) \Lambda_{k}\left(\frac{x-a}{b-a}\right)\right]\right|}\right) \\
& \left.\times \int_{a}^{b} E_{2 n}(x)\left|\varphi^{(2 n)}(x)\right|\right) d x \\
& \times g\left(\frac{\left|\varphi(x)-\sum_{k=0}^{n-1}(b-a)^{2 k}\left[\varphi^{(2 k)}(a) \Lambda_{k}\left(\frac{b-x}{b-a}\right)+\varphi^{(2 k)}(b) \Lambda_{k}\left(\frac{x-a}{b-a}\right)\right]\right|}{E_{2 n}(x)}\right) \\
& \times f\left(\left|\frac{\varphi^{(2 n)}(x)}{m^{(2 n)}(x)}\right|\right) d x
\end{aligned}
$$

holds, where $E_{2 n}$ is Euler polynomial.

Proof. By Widder's lemma we can represent every function $\varphi \in C^{(2 n)}([a, b])$ in the form:

$$
\begin{aligned}
\varphi(x) & =\sum_{k=0}^{n-1}(b-a)^{2 k}\left[\varphi^{(2 k)}(a) \Lambda_{k}\left(\frac{b-x}{b-a}\right)+\varphi^{(2 k)}(b) \Lambda_{k}\left(\frac{x-a}{b-a}\right)\right] \\
& +(b-a)^{2 n-1} \int_{a}^{b} G_{n}\left(\frac{x-a}{b-a}, \frac{s-a}{b-a}\right) \varphi^{(2 n)}(s) d s .
\end{aligned}
$$

Let

$$
\begin{aligned}
\varphi(x) & -\sum_{k=0}^{n-1}(b-a)^{2 k}\left[\varphi^{(2 k)}(a) \Lambda_{k}\left(\frac{b-x}{b-a}\right)+\varphi^{(2 k)}(b) \Lambda_{k}\left(\frac{x-a}{b-a}\right)\right] \\
& =(b-a)^{2 n-1} \int_{a}^{b} G_{n}\left(\frac{x-a}{b-a}, \frac{s-a}{b-a}\right) \varphi^{(2 n)}(s) d s
\end{aligned}
$$

Now apply Theorem 2.1 with $k(x, s)=(b-a)^{2 n-1} G_{n}\left(\frac{x-a}{b-a}, \frac{s-a}{b-a}\right), v(s)=$ $\varphi^{(2 n)}(s)$. Then

$$
K(x)=(b-a)^{2 n-1} \int_{a}^{b} G_{n}\left(\frac{x-a}{b-a}, \frac{s-a}{b-a}\right) d s=E_{2 n}(x),
$$


where $E_{2 n}$ is Euler polynomial.

$$
\begin{aligned}
& u(x)=(b-a)^{2 n-1} \int_{a}^{b} G_{n}\left(\frac{x-a}{b-a}, \frac{s-a}{b-a}\right) \varphi^{(2 n)}(s) d s \\
& =\varphi(x)-\sum_{k=0}^{n-1}(b-a)^{2 k}\left[\varphi^{(2 k)}(a) \Lambda_{k}\left(\frac{b-x}{b-a}\right)+\varphi^{(2 k)}(b) \Lambda_{k}\left(\frac{x-a}{b-a}\right)\right] \\
& m(x)=(b-a)^{2 n-1} \int_{a}^{b} G_{n}\left(\frac{x-a}{b-a}, \frac{s-a}{b-a}\right) m^{(2 n)}(s) d s \\
& =m(x)-\sum_{k=0}^{n-1}(b-a)^{2 k}\left[m^{(2 k)}(a) \Lambda_{k}\left(\frac{b-x}{b-a}\right)+m^{(2 k)}(b) \Lambda_{k}\left(\frac{x-a}{b-a}\right)\right]
\end{aligned}
$$

and inequality (2.3) becomes (2.13) and the proof is complete.

We continue with the following special case of inequality (2.13).

Remark 2.4. If $\varphi^{(2 k)}(a)=\varphi^{(2 k)}(b)=0$, then (2.13) reduces to

$$
\begin{aligned}
& \int_{a}^{b}\left|m(x)-\sum_{k=0}^{n-1}(b-a)^{2 k}\left[m^{(2 k)}(a) \Lambda_{k}\left(\frac{b-x}{b-a}\right)+m^{(2 k)}(b) \Lambda_{k}\left(\frac{x-a}{b-a}\right)\right]\right| \\
& \times f\left(\frac{\mid \varphi(x \mid}{\left|m(x)-\sum_{k=0}^{n-1}(b-a)^{2 k}\left[m^{(2 k)}(a) \Lambda_{k}\left(\frac{b-x}{b-a}\right)+m^{(2 k)}(b) \Lambda_{k}\left(\frac{x-a}{b-a}\right)\right]\right|}\right) \\
& \times g\left(\left|\varphi^{(2 n)}(x)\right|\right) d x \leq \int_{a}^{b} E_{2 n}(x)\left|m^{(2 n)}(x)\right| g\left(\frac{|\varphi(x)|}{E_{2 n}(x)}\right) f\left(\left|\frac{\varphi^{(2 n)}(x)}{m^{(2 n)}(x)}\right|\right) d x .
\end{aligned}
$$

Remark 2.5. If $n(x)=1$, then $m(x)=K(x)$ and (2.13) reduces to

$$
\begin{aligned}
& \int_{a}^{b} E_{2 n}(x) \\
& \times f\left(\frac{\left|\varphi(x)-\sum_{k=0}^{n-1}(b-a)^{2 k}\left[\varphi^{(2 k)}(a) \Lambda_{k}\left(\frac{b-x}{b-a}\right)+\varphi^{(2 k)}(b) \Lambda_{k}\left(\frac{x-a}{b-a}\right)\right]\right|}{E_{2 n}(x)}\right) \\
& \quad \times g\left(\left|\varphi^{(2 n)}(x)\right|\right) d x \leq \int_{a}^{b} E_{2 n}(x) \\
& \times g\left(\frac{\left|\varphi(x)-\sum_{k=0}^{n-1}(b-a)^{2 k}\left[\varphi^{(2 k)}(a) \Lambda_{k}\left(\frac{b-x}{b-a}\right)+\varphi^{(2 k)}(b) \Lambda_{k}\left(\frac{x-a}{b-a}\right)\right]\right|}{E_{2 n}(x)}\right) \\
& \quad \times f\left(\left|\varphi^{(2 n)}(x)\right|\right) d x
\end{aligned}
$$

that is given in [4]. 
We continue with the Hermite interpolating polynomial for the two-point Taylor conditions see [2].

Lemma 2.2. Let $\varphi \in C^{n}[a, b],(n \geq 2, n=2 \mathbf{m})$ then holds

$$
\begin{array}{r}
\varphi(x)=\sum_{i=0}^{\mathbf{m}-1} \sum_{k=0}^{\mathbf{m}-1-i}\left(\begin{array}{c}
\mathbf{m}+k-1 \\
k
\end{array}\right) \cdot\left[\varphi^{(i)}(a) \tau_{i}(x)+\varphi^{(i)}(b) \nu_{i}(x)\right] \\
+\int_{a}^{b} \varphi^{(2 \mathbf{m})}(s) G_{2 T}(x, s) d s
\end{array}
$$

where $\tau_{i}$ and $\nu_{i}$ are defined on $[a, b]$ :

$$
\begin{aligned}
& \tau_{i}(x)=\frac{(x-a)^{i}}{i !}\left(\frac{x-b}{a-b}\right)^{\mathbf{m}}\left(\frac{x-a}{b-a}\right)^{k}, \\
& \nu_{i}(x)=\frac{(x-b)^{i}}{i !}\left(\frac{x-a}{b-a}\right)^{\mathbf{m}}\left(\frac{x-b}{a-b}\right)^{k}
\end{aligned}
$$

$G_{2 T}$ is the Green's function of the two-point Taylor problem:

$G_{2 T}(t, s)= \begin{cases}\frac{(-1)^{\mathbf{m}}}{(2 \mathbf{m}-1) !} p^{\mathbf{m}}(t, s) \sum_{j=0}^{\mathbf{m}-1}\left(\begin{array}{c}\mathbf{m}-1+j \\ j\end{array}\right)(t-s)^{\mathbf{m}-1-j} q^{j}(t, s), & s \leq t \\ \frac{(-1)^{\mathbf{m}}}{(2 \mathbf{m}-1) !} q^{\mathbf{m}}(t, s) \sum_{j=0}^{\mathbf{m}-1}\left(\begin{array}{c}\mathbf{m}-1+j \\ j\end{array}\right)(s-t)^{\mathbf{m}-1-j} p^{j}(t, s), & s \geq t\end{cases}$

and

$$
p(t, s)=\frac{(s-a)(b-t)}{b-a}, q(t, s)=p(s, t), \forall t, s \in[a, b] .
$$

Now, we give a special case of Theorem 1.1 that involves Hermite interpolating polynomial for two-point Taylor conditions.

Corollary 2.3. Let $\varphi:[a, b] \rightarrow \mathbb{R}$ be of $C^{(2 \mathbf{m})}$ on $[a, b]$ such that $\varphi^{(2 \mathbf{m})}$ is either non-negative or non-positive function. Let $m:[a, b] \rightarrow \mathbb{R}$ be of $C^{(2 \mathbf{m})}$ on $[a, b]$ such that $m^{(2 \mathbf{m})}$ is either non-negative or non-positive function. If $f$ is a positive convex function and $g$ a positive concave function on an interval $I \subseteq \mathbb{R}$, then the inequality

$$
\begin{aligned}
& \left.\int_{a}^{b} \mid m(x)-\sum_{i=0}^{\mathbf{m}-1} \sum_{k=0}^{\mathbf{m}-1-i}\left(\begin{array}{c}
\mathbf{m}+k-1 \\
k
\end{array}\right)\left[m^{(i)}(a) \tau_{i}(x)+m^{(i)}(b) \nu_{i}(x)\right]\right) \mid \\
& \times f\left(\frac{\left|\varphi(x)-\sum_{i=0}^{\mathbf{m}-1} \sum_{k=0}^{\mathbf{m}-1-i}\left(\begin{array}{c}
\mathbf{m}+k-1 \\
k
\end{array}\right)\left[\varphi^{(i)}(a) \tau_{i}(x)+\varphi^{(i)}(b) \nu_{i}(x)\right]\right|}{\left.\mid m(x)-\sum_{i=0}^{\mathbf{m}-1} \sum_{k=0}^{\mathbf{m}-1-i}\left(\begin{array}{c}
\mathbf{m}+k-1 \\
k
\end{array}\right)\left[m^{(i)}(a) \tau_{i}(x)+m^{(i)}(b) \nu_{i}(x)\right]\right) \mid}\right) \\
& \times g\left(\left|\varphi^{(2 \mathbf{m})}(x)\right|\right) d x \leq \int_{a}^{b} \frac{1}{(2 \mathbf{m}) !}(x-a)^{\mathbf{m}}(b-x)^{\mathbf{m}}\left|m^{(2 \mathbf{m})}(x)\right| f\left(\left|\frac{\varphi^{(2 \mathbf{m})}(x)}{m^{(2 \mathbf{m})}(x)}\right|\right)
\end{aligned}
$$


$\times g\left(\frac{(2 \mathbf{m}) !\left|\varphi(x)-\sum_{i=0}^{\mathbf{m}-1} \sum_{k=0}^{\mathbf{m}-1-i}\left(\begin{array}{c}\mathbf{m}+k-1 \\ k\end{array}\right)\left[\varphi^{(i)}(a) \tau_{i}(x)+\varphi^{(i)}(b) \nu_{i}(x)\right]\right|}{(x-a)^{\mathbf{m}}(b-x)^{\mathbf{m}}}\right) d x$

holds.

Proof. Since $G_{2 T}(t, s)$ is a symmetric function, we define

$$
\begin{array}{r}
\varphi(x)-\sum_{i=0}^{\mathbf{m}-1} \sum_{k=0}^{\mathbf{m}-1-i}\left(\begin{array}{c}
\mathbf{m}+k-1 \\
k
\end{array}\right) \cdot\left[\varphi^{(i)}(a) \tau_{i}(x)+\varphi^{(i)}(b) \nu_{i}(x)\right] \\
=\int_{a}^{b} \varphi^{(2 \mathbf{m})}(s) G_{2 T}(x, s) d s .
\end{array}
$$

Now apply Theorem 2.1 with $k(x, s)=G_{2 T}(x, s), v(s)=\varphi^{(2 \mathbf{m})}(s), n(x)=$ $m^{(2 \mathbf{m})}(x)$. We obtain

$$
\begin{aligned}
|K(x)| & =\int_{a}^{b}\left|G_{2 T}(x, s)\right| d s=\frac{1}{(2 \mathbf{m}) !}\left|(x-a)^{\mathbf{m}}(x-b)^{\mathbf{m}}\right|=\frac{1}{(2 \mathbf{m}) !}(x-a)^{\mathbf{m}}(b-x)^{\mathbf{m}}, \\
u(x) & =\int_{a}^{b} G_{2 T}(x, s) \varphi^{(2 \mathbf{m})}(s) d s \\
& =\varphi(x)-\sum_{i=0}^{\mathbf{m}-1} \sum_{k=0}^{\mathbf{m}-1-i}\left(\begin{array}{c}
\mathbf{m}+k-1 \\
k
\end{array}\right) \cdot\left[\varphi^{(i)}(a) \tau_{i}(x)+\varphi^{(i)}(b) \nu_{i}(x)\right]
\end{aligned}
$$

and inequality (1.4) becomes (2.21), so the proof is complete.

Remark 2.6. If $\varphi^{(i)}(a)=\varphi^{(i)}(b)=0$, then inequality (2.21) reduces to

$$
\begin{aligned}
& \left.\int_{a}^{b} \mid m(x)-\sum_{i=0}^{\mathbf{m}-1} \sum_{k=0}^{\mathbf{m}-1-i}\left(\begin{array}{c}
\mathbf{m}+k-1 \\
k
\end{array}\right)\left[m^{(i)}(a) \tau_{i}(x)+m^{(i)}(b) \nu_{i}(x)\right]\right) \mid \\
& \times f\left(\frac{|\varphi(x)|}{\left.\mid m(x)-\sum_{i=0}^{\mathbf{m}-1} \sum_{k=0}^{\mathbf{m}-1-i}\left(\begin{array}{c}
\mathbf{m}+k-1 \\
k
\end{array}\right)\left[m^{(i)}(a) \tau_{i}(x)+m^{(i)}(b) \nu_{i}(x)\right]\right)}\right) \\
& \times g\left(\left|\varphi^{(2 \mathbf{m})}(x)\right|\right) d x \leq \int_{a}^{b} \frac{1}{(2 \mathbf{m}) !}(x-a)^{\mathbf{m}}(b-x)^{\mathbf{m}}\left|m^{(2 \mathbf{m})}(x)\right| \\
& \times f\left(\left|\frac{\varphi^{(2 \mathbf{m})}(x)}{m^{(2 \mathbf{m})}(x)}\right|\right) g\left(\frac{(2 \mathbf{m}) !|\varphi(x)|}{(x-a)^{\mathbf{m}}(b-x)^{\mathbf{m}}}\right) d x .
\end{aligned}
$$


Remark 2.7. If $n(x)=1$, then $m(x)=K(x)$ and (2.21) reduces to

$$
\begin{aligned}
& \int_{a}^{b}(x-a)^{\mathbf{m}}(b-x)^{\mathbf{m}} \\
& \times f\left(\frac{(2 \mathbf{m}) !\left|\varphi(x)-\sum_{i=0}^{\mathbf{m}-1} \sum_{k=0}^{\mathbf{m}-1-i}\left(\begin{array}{c}
\mathbf{m}+k-1 \\
k
\end{array}\right)\left[\varphi^{(i)}(a) \tau_{i}(x)+\varphi^{(i)}(b) \nu_{i}(x)\right]\right|}{(x-a)^{\mathbf{m}}(b-x)^{\mathbf{m}}}\right) \\
& \quad \times g\left(\left|\varphi^{(2 \mathbf{m})}(x)\right|\right) d x \\
& \leq \int_{a}^{b}(x-a)^{\mathbf{m}}(b-x)^{\mathbf{m}} \\
& \times g\left(\frac{(2 \mathbf{m}) !\left|\varphi(x)-\sum_{i=0}^{\mathbf{m}-1} \sum_{k=0}^{\mathbf{m}-1-i}\left(\begin{array}{c}
\mathbf{m}+k-1 \\
k
\end{array}\right)\left[\varphi^{(i)}(a) \tau_{i}(x)+\varphi^{(i)}(b) \nu_{i}(x)\right]\right|}{(x-a)^{\mathbf{m}}(b-x)^{\mathbf{m}}}\right) \\
& \quad \times f\left(\left|\varphi^{(2 \mathbf{m})}(x)\right|\right) d x .
\end{aligned}
$$

that is given in [4].

Acknowledgement. This work has been supported by Croatian Science Foundation under the project 5435.

\section{REFERENCES}

[1] M. Andrić, J. Pečarić, I. Perić, A multiple Opial type inequality for the RiemannLiouville fractional derivatives, J. Math. Inequal., 7 (1) (2013), 139-150.

[2] R. P. Agarwal and P. J. Y. Wong, Error Inequalities in Polynomial Interpolation and Their Applications, Kluwer Academic Publishers, Dordrecht / Boston / London, 1993.

[3] R. P. Agarwal and P. Y. H. Pang, Opial Inequalities with Applications in Differential and Difference Equations, Kluwer Academic Publishers, Dordrecht, Boston, London, 1995.

[4] A. Barbir, K. Krulić Himmelreich and J. Pečarić, General Opial type inequality, Aequationes Math., 89 (3) (2015), 641-655.

[5] Z. Opial, Sur une inéqalité, Ann. Polon. Math., 8 (1960), 29-32.

[6] J. M. Whittaker, On Lidstone series and two-point expansions of analytic functions, Proc. Lond. Math. Soc., 36 (1933-1934), 451-469.

[7] D. V. Widder, Completly convex function and Lidstone series, Trans. Am. Math. Soc., 51 (1942), 387-398.

(Received: July 1, 2015)

Ana Barbir

Faculty of Civil Engineering and

Architecture

Matice hrvatske 15

21000 Split, Croatia

abarbir@gradst.hr 
Kristina Krulić Himmelreich Faculty of Textile Technology University of Zagreb

Prilaz baruna Filipovića 28a 10000 Zagreb, Croatia

kkrulic@ttf.hr

Josip Pečarić

Faculty of Textile Technology

University of Zagreb

Prilaz baruna Filipovića 28a

10000 Zagreb, Croatia

pecaric@element.hr 